\title{
Trial Equation Method for Solving the Improved Boussinesq Equation
}

\author{
Yang Li \\ Department of Mathematics, Northeast Petroleum University, Daqing, China \\ Email: liyang120918@163.com
}

Received January 10, 2014; revised February 10, 2014; accepted February 17, 2014

Copyright (c) 2014 Yang Li. This is an open access article distributed under the Creative Commons Attribution License, which permits unrestricted use, distribution, and reproduction in any medium, provided the original work is properly cited. In accordance of the Creative Commons Attribution License all Copyrights (C) 2014 are reserved for SCIRP and the owner of the intellectual property Yang Li. All Copyright (C) 2014 are guarded by law and by SCIRP as a guardian.

\section{ABSTRACT}

Trial equation method is a powerful tool for obtaining exact solutions of nonlinear differential equations. In this paper, the improved Boussinesq is reduced to an ordinary differential equation under the travelling wave transformation. Trial equation method and the theory of complete discrimination system for polynomial are used to establish exact solutions of the improved Boussinesq equation.

\section{KEYWORDS}

The Nonlinear Partial Differential Equation; Complete Discrimination System for Polynomial; Trial Equation Method; Traveling Wave Transform; The Improved Boussinesq Equation

\section{Introduction}

In every field of engineering technology, science research, natural world and human society activities, nonlinear phenomena occupy an important position. The investigation of exact solutions of nonlinear evolution equations helps us understand these phenomena better. With the development of soliton theory and the application of computer symbolic system such as Matlab and Mathematica, many powerful methods for obtaining exact solutions of nonlinear evolution equations are presented, such as the inverse scattering method [1], Hirotas bilinear transformation [2,3], the tanh method [4], sine-cosine method [5], homogeneous balance method [6,7], expfunction method [8], and so on. Recently, Professor Liu proposed a powerful method named trial equation method [9-11] for finding exact solutions to nonlinear differential equations.

In coastal engineering, Boussinesq-type equations are frequently used in computer models for the simulation of water waves in shallow seas and harbours. The objective of this paper is to apply Liu's method and the theory of complete discrimination system for polynomial [12-16] to find the exact solutions of the nonlinear differential equation.

\section{Description of Trial Equation Method}

The objective of this section is to outline the use of trial equation method for solving a nonlinear partial differential equation (PDE). Suppose we have a nonlinear PDE for $u(x, t)$ in the form

$$
P\left(u, u_{x}, u_{t}, u_{x x}, u_{t t}, u_{x t}, \cdots\right)=0
$$

where $P$ is a polynomial, which includes nonlinear terms and the highest order derivatives and so on.

Step 1 Taking the wave transformation $u=u\left(\xi_{1}\right), \xi_{1}=k x+\omega t$, reduces Equation (1) to the ordinary differential equation (ODE).

$$
M\left(u, k u^{\prime}, \omega u^{\prime}, k^{2} u^{\prime \prime}, \omega^{2} u^{\prime \prime}, k \omega u^{\prime \prime}, \cdots\right)=0
$$


Step 2 Take trial equation method

$$
u^{\prime \prime}=F(u)=a_{0}+a_{1} u+\cdots+a_{m} u^{m} .
$$

Integrating the Equation (3) with respect to $\xi_{1}$ once, we get

$$
\left(u^{\prime}\right)^{2}=H(u)=\frac{2 a_{m}}{m+1} u^{m+1}+\cdots+a_{1} u^{2}+2 a_{0} u+d
$$

where $\mathrm{m}, a_{i}$ and integration constant $d$ are to be determined. Substituting Equations (3), (4) and other derivative terms into Equation (2) yields a polynomial $G(u)$ of $\mu$. According to the balance principle we can determine the value of $m$. Setting the coefficients of $G(u)$ to zero, we get a system of algebraic equations. Solving this system, we can determine values of $a_{o}, a_{1}, \cdots, a_{m}$ and integration constant.

Step 3 Rewrite Equation (4) by the integral form

$$
\pm\left(\xi-\xi_{0}\right)=\int \frac{\mathrm{d} u}{\sqrt{H(u)}} .
$$

According to the complete discrimination system of the polynomial, we classify the roots of $H(u)$ and solve the integral Equation (5). Thus we obtain the exact solutions to Equation (1).

\section{Application of Trial Equation Method}

The improved Boussinesq equation $[17,18]$ reads as

$$
u_{t t}-u_{x x}-u u_{x x}-\left(u_{x}\right)^{2}-u_{x x t t}=0 .
$$

Taking the traveling wave transformation $u=u\left(\xi_{1}\right)$ and $\xi_{1}=k x+\omega t$, we can obtain the corresponding reduced ODE.

$$
\left(\omega^{2}-k^{2}\right) u^{\prime \prime}-k^{2} u u^{\prime \prime}-k^{2}\left(u^{\prime}\right)^{2}-k^{2} \omega^{2} u^{\prime \prime \prime}=0 .
$$

we take the trial equation as follows

$$
u^{\prime \prime}=a_{0}+a_{1} u+\cdots+a_{m} u^{m} .
$$

According to the trial equation method of rank homogeneous equation, balancing $u^{\prime \prime \prime}$ with $u u^{\prime \prime}$ (or $\left(u^{\prime}\right)^{2}$ ) gets $m=2$, so Equation (8) has the following specific form

$$
u^{\prime \prime}=a_{0}+a_{1} u+a_{2} u^{2} .
$$

Integrating Equation (9) with respect to $\xi_{1}$ once, we yield

$$
\left(u^{\prime}\right)^{2}=\frac{2}{3} a_{2} u^{3}+a_{1} u^{2}+2 a_{0} u+d .
$$

where values of $a_{o}, a_{1} a_{2}$ and the integration constant $d$ are to be determined latter. By Equation (9) and Equation (10), we derive the following formula

$$
u^{\prime \prime \prime}=\frac{10}{3} a_{2}^{2} u^{3}+5 a_{1} a_{2} u^{2}+\left(6 a_{0} a_{2}+a_{1}^{2}\right) u+a_{0} a_{1}+2 a_{2} d .
$$

Substituting Equations (9), (10) and (11) into Equation (7), we have

where

$$
r_{3} u^{3}+r_{2} u^{2}+r_{1} u+r_{0}=0
$$

$$
\begin{gathered}
r_{0}=\omega^{2} a_{0}-k^{2} d-k^{2} a_{0}-k^{2} \omega^{2}\left(a_{0} a_{1}+2 a_{2} d\right) . \\
r_{1}=-3 k^{2} a_{0}-k^{2} a_{1}+\omega^{2} a_{1}-k^{2} \omega^{2}\left(a_{1}^{2}+6 a_{0} a_{2}\right) . \\
r_{2}=-2 k^{2} a_{1}-k^{2} a_{2}+\omega^{2} a_{2}-5 k^{2} \omega^{2} a_{1} a_{2} . \\
r_{3}=-\frac{5}{3} k^{2} a_{2}-\frac{10}{3} k^{2} \omega^{2} a_{2}^{2} .
\end{gathered}
$$


Let the coefficient $r_{i}=0(i=0,1,2,3)$ be zero, we will yield nonlinear algebraic equations. Solving the equations, we will determine the values of $a_{0}, a_{1}, a_{2}, d$. We get $a_{1}=\frac{1}{k^{2}}-\frac{1}{\omega^{2}}, a_{2}=-\frac{1}{2 \omega^{2}}, a_{0}$ and $d$ are two arbitrary constants. When the above conditions are satisfied, we use the complete discrimination system for the third order polynomial and have the following solving process.

Let

$$
v=\left(\frac{2}{3} a_{2}\right)^{\frac{1}{3}} u, \xi=\left(\frac{2}{3} a_{2}\right)^{\frac{1}{3}} \xi_{1}, d_{2}=a_{1}\left(\frac{2}{3} a_{2}\right)^{-\frac{2}{3}}, d_{1}=2 a_{0}\left(\frac{2}{3} a_{2}\right)^{-\frac{1}{3}}, d_{0}=d .
$$

Then Equation (10) becomes

$$
\left(v^{\prime}\right)^{2}=v^{3}+d_{2} v^{2}+d_{1} v+d_{0} .
$$

where $v$ is a function of $\xi$. The integral form of Equation (18) is

$$
\pm\left(\xi-\xi_{0}\right)=\int \frac{\mathrm{d} v}{\sqrt{v^{3}+d_{2} v^{2}+d_{1} v+d_{0}}} .
$$

Denote

$$
\begin{gathered}
F(v)=v^{3}+d_{2} v^{2}+d_{1} v+d_{0} . \\
\Delta=-27\left(\frac{2 d_{2}^{3}}{27}+d_{0}-\frac{d_{1} d_{3}}{3}\right)^{2}-4\left(d_{1}-d_{2}^{2}\right)^{3}, D_{1}=d_{1}-\frac{d_{2}^{2}}{3} .
\end{gathered}
$$

According to the complete discrimination system, we give the corresponding single traveling wave solutions to Equation (6).

Case1. $\Delta=0, D_{1}<0 . F(v)=0$ has a double real root and a simple real root. Then we have

$$
F(v)=\left(v-\lambda_{1}\right)^{2}\left(v-\lambda_{2}\right), \lambda_{1} \neq \lambda_{2} .
$$

When $v>\lambda_{2}$, the corresponding solutions are

$$
\begin{aligned}
& u_{1}=\left(\frac{2}{3} a_{2}\right)^{-\frac{1}{3}}\left\{\left(\lambda_{1}-\lambda_{2}\right) \tanh ^{2}\left[\frac{\sqrt{\lambda_{1}-\lambda_{2}}}{2}\left(\frac{2}{3} a_{2}\right)^{\frac{1}{3}}\left(k x+\omega t-\xi_{0}\right)\right]+\lambda_{2}\right\},\left(\lambda_{1}>\lambda_{2}\right) ; \\
& u_{2}=\left(\frac{2}{3} a_{2}\right)^{-\frac{1}{3}}\left\{\left(\lambda_{1}-\lambda_{2}\right) \operatorname{coth}^{2}\left[\frac{\sqrt{\lambda_{1}-\lambda_{2}}}{2}\left(\frac{2}{3} a_{2}\right)^{\frac{1}{3}}\left(k x+\omega t-\xi_{0}\right)\right]+\lambda_{2}\right\},\left(\lambda_{1}>\lambda_{2}\right) ; \\
& u_{3}=\left(\frac{2}{3} a_{2}\right)^{-\frac{1}{3}}\left\{\left(-\lambda_{1}+\lambda_{2}\right) \sec ^{2}\left[\frac{\sqrt{-\lambda_{1}+\lambda_{2}}}{2}\left(\frac{2}{3} a_{2}\right)^{\frac{1}{3}}\left(k x+\omega t-\xi_{0}\right)\right]+\lambda_{1}\right\},\left(\lambda_{1}<\lambda_{2}\right) .
\end{aligned}
$$

Case2. $\Delta=0, D_{1}=0 . F(v)=0$ has a triple root. Then we have

$$
F(v)=(v-\lambda)^{3} .
$$

The corresponding solution is

$$
u_{4}=4\left(\frac{2}{3} a_{2}\right)^{-\frac{2}{3}}\left(k x+\omega t-\xi_{0}\right)^{-2}+\lambda .
$$

Case 3. $\Delta>0, D_{1}<0 . F(v)=0$ has three different real roots. Then we have

$$
F(v)=\left(v-\lambda_{1}\right)\left(v-\lambda_{2}\right)\left(v-\lambda_{3}\right), \lambda_{1}<\lambda_{2}<\lambda_{3} .
$$

When $\lambda_{1}<v<\lambda_{2}$, we take the transformation as follows 


$$
v=\lambda_{1}+\left(\lambda_{2}-\lambda_{1}\right) \sin ^{2} \varphi
$$

According to the Equation (19), we have

$$
\pm\left(\xi-\xi_{0}\right)=\int \frac{\mathrm{d} v}{\sqrt{F(v)}}=\frac{2}{\sqrt{\lambda_{3}-\lambda_{1}}} \int \frac{\mathrm{d} \varphi}{\sqrt{1-m^{2} \sin ^{2} \varphi}} .
$$

where $m^{2}=\frac{\lambda_{2}-\lambda_{1}}{\lambda_{3}-\lambda_{1}}$. On the basis of Equation (30) and the definition of the Jacobi elliptic sine function, we have

$$
v=\lambda_{1}+\left(\lambda_{2}-\lambda_{1}\right) \operatorname{sn}^{2}\left(\frac{\sqrt{\lambda_{3}-\lambda_{1}}}{2}\left(\xi_{1}-\xi_{0}\right), m\right) .
$$

The corresponding solutions is

$$
u_{5}=\left(\frac{2}{3} a_{2}\right)^{-\frac{1}{3}}\left[\lambda_{1}+\left(\lambda_{2}-\lambda_{1}\right) \operatorname{sn}^{2}\left(\frac{\sqrt{\lambda_{3}-\lambda_{1}}}{2}\left(\frac{2}{3} a_{2}\right)^{\frac{1}{3}}\left(k x+\omega t-\xi_{0}\right), m\right)\right] .
$$

when $v>\lambda_{3}$, we take the transformation as follows

$$
v=\frac{-\lambda_{2} \sin ^{2} \varphi+\lambda_{3}}{\cos ^{2} \varphi}
$$

The corresponding solutions is

$$
u_{6}=\left(\frac{2}{3} a_{2}\right)^{-\frac{1}{3}}\left[\frac{\lambda_{3}-\lambda_{2} \operatorname{sn}^{2}\left(\frac{\sqrt{\lambda_{3}-\lambda_{1}}}{2}\left(\frac{2}{3} a_{2}\right)^{\frac{1}{3}}\left(k x+\omega t-\xi_{0}\right), m\right)}{\operatorname{cn}^{2}\left(\frac{\sqrt{\lambda_{3}-\lambda_{1}}}{2}\left(\frac{2}{3} a_{2}\right)^{\frac{1}{3}}\left(k x+\omega t-\xi_{0}\right), m\right)}\right] .
$$

where $m^{2}=\frac{\lambda_{2}-\lambda_{1}}{\lambda_{3}-\lambda_{1}}$

Case 4. $\Delta<0 . F(v)=0$ has only a real root. Then we have

$$
F(v)=(v-\lambda)\left(v^{2}+p v+q\right), p^{2}-4 q<0 .
$$

when $v>\lambda_{1}$, we take the transformation as follows

$$
v=\lambda+\sqrt{\lambda^{2}+p \lambda+q} \tan ^{2} \frac{\varphi}{2} .
$$

According to the Equation (19), we have

where $m^{2}=\frac{1}{2}\left(1-\frac{\lambda+\frac{p}{2}}{\sqrt{\lambda^{2}+p \lambda+q}}\right)$. On the basis of Equation (37) and the definition of the Jacobi elliptic cosine function, we have

$$
v=\lambda+\frac{2 \sqrt{\lambda^{2}+p \lambda+q}}{1+\mathrm{cn}\left(\left(\lambda^{2}+p \lambda+q\right)^{\frac{1}{4}}\left(\xi_{1}-\xi_{0}\right), m\right)}-\sqrt{\lambda^{2}+p \lambda+q} .
$$

The corresponding solutions is 


$$
u_{7}=\left(\frac{2}{3} a_{2}\right)^{-\frac{1}{3}}\left[\lambda+\frac{2 \sqrt{\lambda^{2}+p \lambda+q}}{1+\mathrm{cn}\left(\left(\lambda^{2}+p \lambda+q\right)^{\frac{1}{4}}\left(\frac{2}{3} a_{2}\right)^{\frac{1}{3}}\left(k x+\omega t-\xi_{0}\right), m\right)}-\sqrt{\lambda^{2}+p \lambda+q}\right] .
$$

In Equations (23), (24), (25), (27), (32), (34), and (39), the integration constant $\xi_{0}$ has been rewritten, but we still use it. The solutions $u_{i}(i=1, \cdots, 7)$ are all possible exact traveling wave solutions to Equation (6). We can see it is easy to write the corresponding solutions to the improved Boussinesq equation.

\section{Conclusion}

Trial equation method is a systematic method to solve nonlinear differential equations. The advantage of this method is that we can deal with nonlinear equations with linear methods. This method has the characteristics of simple steps and clear effectivity. Based on the idea of the trial equation method and the aid of the computerized symbolic computation, some exact traveling wave solutions to the improved Boussinesq equation have been obtained. With the same method, some of other equations can be dealt with.

\section{Acknowledgements}

I would like to thank the referees for their valuable suggestions.

\section{REFERENCES}

[1] M. J. Ablowitz and P. A. Clarson, "Solitons, Nonlinear Evolution Equations and Inverse Scattering," Cambridge University Press, New York, 1991. http://dx.doi.org/10.1017/CBO9780511623998

[2] R. Hirota, “Exact Envelope-Soliton Solutions of a Nonlinear Wave Equation,” Journal of Mathematical Physics, Vol. 14, No. 7, 1973, p. 805. http://dx.doi.org/10.1063/1.1666399

[3] R. Hirota and J. Satsuma, “Soliton Solutions of a Coupled Korteweg-de Vries Equation,” Physical Letters A, Vol. 85, No. 8-9, 1981, pp. 407-408. http://dx.doi.org/10.1016/0375-9601(81)90423-0

[4] E. Fan, "Extended Tank-Function Method and Its Applications to Nonlinear Equations,” Physical Letters A, Vol. 277, No. 4, 2000, pp. 212-218.

[5] C. T. Yan, “A Simple Transformation for Nonlinear Waves,” Physical Letters A, Vol. 224, No. 1-2, 1996, pp. 77-84. http://dx.doi.org/10.1016/S0375-9601(96)00770-0

[6] M. Wang, "Solitary Wave Solutions for Variant Boussinesq Equations,” Physical Letters A, Vol. 199, No. 3, 1995, pp. 169172.

[7] M. L. Wang, “Solitary Wave Solutions for Variant Boussinesq Equations,” Physical Letters A, Vol. 199, No. 3-4, 1995, pp. 169-172. http://dx.doi.org/10.1016/0375-9601(95)00092-H

[8] W. X. Ma and J. H. Lee, "A Transformed Rational Function Method and Exact Solutions to the $3+1$ Dimensional Jimbo-Miwa Equation,” Chaos, Solitons and Fractals, Vol. 42, No. 3, 2009, pp. 1356-1363. http://dx.doi.org/10.1016/j.chaos.2009.03.043

[9] “Applications,” Communications in Theoretical Physics, Vol. 45, No. 2, 2006, pp. 219-223.

[10] C. S. Liu, “Trial Equation Method and Its Applications to Nonlinear Evolution Equations,” Acta Physical Sinica, Vol. 54, 2005, p. 2505 (in Chinese).

[11] C. S. Liu, "Using Trial Equation Method to Solve the Exact Solutions for Two Kinds of KdV Equations with Variable Coefficients,” Acta Physical Sinica, Vol. 54, No. 10, 2005, p. 4506.

[12] C. S. Liu, "Representations and Classification of Traveling Wave Solutions to Sinh-Gördon Equation,” Communications in Theoretical Physics, Vol. 49, 2008, pp. 153-158. http://dx.doi.org/10.1088/0253-6102/49/1/33

[13] C. S. Liu, "Solution of ODE $u^{\prime \prime}+p(u)\left(u^{\prime}\right)^{2}+q(u)=0$ and Applications to Classifications of All Single Travelling Wave Solutions to Some Nonlinear Mathematical Physics Equations," Communications in Theoretical Physics, Vol. 49, 2008, pp. 291-296. http://dx.doi.org/10.1088/0253-6102/49/2/07

[14] C. S. Liu, “Applications of Complete Discrimination System for Polynomial for Classifications of Traveling Wave Solutions to Nonlinear Differential Equations,” Computer Physics Communications, Vol. 181, 2010, pp. 317-324. 
http://dx.doi.org/10.1016/j.cpc.2009.10.006

[15] C. S. Liu, “Classification of All Single Travelling Wave Solutions to Calogero-Degasperis-Focas Equation,” Communications in Theoretical Physics, Vol. 48, 2007, pp. 601-604. http://dx.doi.org/10.1088/0253-6102/48/4/004

[16] C. S. Liu, “All Single Traveling Wave Solutions to Nizhnok-Novikov-Veselov Equation,” Communications in Theoretical Physics, Vol. 45, 2006, pp. 991-992. http://dx.doi.org/10.1088/0253-6102/45/6/006

[17] P. L. Christiansen and V. Muto, “Physica D 68,” 1993. http://dx.doi.org/10.1016/0167-2789(93)90033-W

[18] Z. Yang and B. Y. C. Hon, “An Improved Modified Extended Tanh-Function Method,” Zeitschrift fur Naturforschung AJournal of Physical Sciences, Vol. 61, No. 3-4, 2006, pp. 103-115. 